\title{
Experimental and Theoretical Validation for the Incremental Theory of Diffraction
}

\author{
Danilo Erricolo, Senior Member, IEEE, Stefano Mihai Canta, Student Member, IEEE, \\ Harun Taha Hayvacı , Student Member, IEEE, Matteo Albani, Member, IEEE
}

\begin{abstract}
Experimental and theoretical approaches to verify the validity of the Incremental Theory of Diffraction (ITD) are considered. After providing a simple recipe for the application of the ITD, three geometries are examined for its validation. First, the ITD formulation of the diffraction from a perfect electric conductor (PEC) straight wedge is compared with the Uniform Theory of Diffraction (UTD) and with measurement results. Second, the ITD formulation of the diffraction from a PEC disc is compared with measurement results and with the exact solution of a boundary value problem involving oblate spheroidal functions. Third, the ITD formulation of the diffraction from a hole in a PEC plane is compared with the exact solution of a boundary value problem involving oblate spheroidal functions. In particular, this is the first time that ITD results for diffraction from the disc and hole in a plane geometries are validated using exact solutions computed at a caustic. In all cases examined, very good agreement is found.
\end{abstract}

Index Terms-Geometrical Theory of Diffraction, Incremental Theory of Diffraction, Oblate Spheroidal Functions, Diffraction, Measurements

\section{INTRODUCTION}

$\mathbf{T}$ HE Incremental Theory of Diffraction [1] (ITD) is an extension of the well-known Uniform Theory of Diffraction (UTD)[2]. The UTD removes the singularities at the shadow boundaries present in Keller's Geometrical Theory of Diffraction [3]. The ITD was introduced to remove singularities close to and at caustics in the UTD formulation; it also has the advantages of being reciprocal, being easy to apply to complex contours, and naturally blending to the UTD formulation when the ray regime is well established.

In general, incremental field representation may be used in conjunction with ray-methods to (1) overcome difficulties close to and at caustics; and (2) to improve the field estimate in the transition zones where the ray field regime is not established. Several contributions have been

Erricolo, Canta, and Hayvacı are with the Department of Electrical and Computer Engineering, University of Illinois at Chicago, Chicago, IL 60607 USA. Email: erricolo@ece.uic.edu scanta2@uic.edu, hhayva2@uic.edu.

Albani is with the Dipartimento di Ingegneria dell'Informazione, Università di Siena, Via Roma 56, 53100 Siena, Italy. Email: matteo.albani@ing.unisi.it

This research was supported in part by the DoD/AFOSR MURI grant FA9550-05-1-0443 and in part by the DoD/DARPA grant N00173-06-1-G006 provided in the literature and they may be classified as current-based methods and field-based methods.

Current-based methods are founded on the field representation introduced by the Physical Theory of Diffraction (PTD) [4]. They augment the Physical Optics (PO) field by improving the local estimates of the currents, deduced from local canonical problems, and lead to the Elementary Edge Wave Theory or the Incremental Length Diffraction Coefficient. Methods to localize fringe field contributions include those provided in [5], [6], [7], [8], [9], [10], [11], [12], [13].

The ITD belongs to the category of field-based methods that are intended to directly deduce incremental field contributions from the field of local canonical problems. A general procedure for defining such incremental fields was introduced in [14], [15], [16], which depends on the ability to construct a suitable spatial integral representation of the exact solution for the relevant local canonical problem [17]. As an example, the ITD was applied in [18].

Using simple contour integrations, this theory introduces the concept of incremental contributions to calculate the fields diffracted by points along the contour of an obstacle. The results provided by this theory are in most cases equivalent to those computed using the UTD; the only cases in which they differ are close to and at caustic points. In this latter situation, the ITD provides the correct evaluation of the fields, as it will be shown by comparison with the exact solution of a boundary value problem involving oblate spheroidal functions.

In this paper, the time-convention $\exp (j \omega t)$ is assumed and suppressed throughout. The paper is organized as follows: in Section II, a simple recipe for the practical application of the ITD is described. Section III discusses the diffraction from a PEC straight wedge, evaluated using the ITD, the UTD, and measurement results. In Section IV, the diffraction from a PEC circular disc is examined using the ITD, measurement results, and an exact solution developed in Section V. Section V describes validations with the exact solutions for the disc and a hole in a PEC plane. Conclusions are provided in Section VI.

The contributions of this paper are at least twofold. First, experimental validations for the ITD are provided for the straight wedge and the disc geometries. Second, for the disc geometry and the hole in a plane geometry, comparisons with the exact solutions of boundary value problems show that the ITD provides correct values close to and at caustics. 


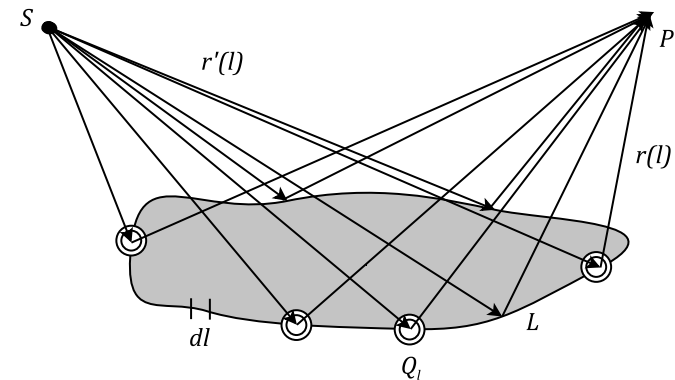

Fig. 1. Incremental contributions from all the points along the curved contour $L$.

\section{Essentials of the ITD}

The application of the ITD is simple and straightforward because it takes care of properly evaluating the diffracted fields by performing a numerical integration, without the need of finding, e.g., stationary phase points. As an example, consider the object shown in Fig. 1. The diffracted field observed at $P$ is the superposition of incremental contributions distributed along the contour $L$. Such contributions are due to the illumination at each local diffraction point $Q_{l}$ by the source at $S$. Because of the locality principle of diffraction at high-frequency, interactions among different incremental contributions are not taken into account, as explained in [1], since they constitute higher order asymptotic contributions. To determine the diffracted field $\mathbf{E}^{\mathbf{d}}(P)$, the ITD only requires the evaluation of the following line integral

$$
\mathbf{E}^{\mathbf{d}}(P)=\int_{L} d \mathbf{E}^{\mathbf{d}}=\int_{L} \overline{\mathbf{D}}(l) \mathbf{E}_{\mathbf{0}}(l) \frac{e^{-j k r(l)}}{r(l)} d l,
$$

where $d \mathbf{E}^{\mathbf{d}}$ is the vector incremental contribution, $\overline{\mathbf{D}}(l)$ is the dyadic that contains the incremental diffraction coefficients, $\mathbf{E}_{\mathbf{0}}(l)$ the incident field upon the point of integration, $r(l)$ the distance from the integration position to the observation point, and $k$ is the free space wavenumber. The relative importance of each incremental contribution is taken care of by the dyadic $\overline{\mathbf{D}}(l)$ and, overall, by the numerical integration. Equation (1), provides a high frequency asymptotic expression for the diffracted field that only retains the leading term. Terms of order $(k r)^{-n}$, with $n>1$, are neglected.

Clearly, to compute the total field $\mathbf{E}(P)$ one needs to add the geometrical optics contributions, i.e. the incident $\mathbf{E}^{\mathbf{i}}$ and reflected $\mathbf{E}^{\mathbf{r}}$ fields, to the ITD diffracted field $\mathbf{E}^{\mathbf{d}}$ obtained from (1). The advantages of the ITD include

- It is simple to apply to complex contours, as it requires only the evaluation of (1).

- The minimum radius of curvature $a$ of a contour should, in principle, satisfy $a \gg \lambda$, but in practice good results for engineering applications are obtained when $a \approx \lambda$.

- It satisfies reciprocity, which is embedded into Maxwell's equations.

- It naturally blends into the UTD solution when the ray regime is well established.
- When combined with geometrical optics (GO) to compute the total field, it is computationally faster than the combination of PO and PTD.

The main limitation of the present version of the ITD [1] is that it is affected by the caustics of GO and that GO discontinuities are not exactly compensated for astigmatic wavefronts. These are encountered when dealing with astigmatic sources or with wavefronts reflected by scattering objects with curved surfaces. Hence, in the present version, the ITD can be effectively applied only to objects with flat faces of arbitrary contours. Therefore, when compared to PO+PTD, the GO+ITD approach is, roughly speaking, less general but more efficient. Namely, GO+ITD can be applied only to describe flat surfaces illuminated by non astigmatic sources and it is therefore less general than PO+PTD that can also manage curved surfaces and astigmatic illuminating wavefronts. However, by resorting to GO, GO+ITD does not require a PO surface integration but only a line integration on the edges, consequently it is, in most cases, less time consuming.

All one needs to do is to define an appropriate coordinate system and all pertinent geometrical quantities.

\section{A. Recipe for the application of the ITD}

\begin{tabular}{|c|c|}
\hline$\hat{\mathbf{x}}_{\text {loc }}$ & $\begin{array}{l}\text { u.v. tangent to the face and normal to the } \\
\text { edge of the contour at } Q_{l}\end{array}$ \\
\hline$\hat{\mathbf{y}}_{\text {loc }}$ & $\begin{array}{c}\text { u.v. normal to the face and normal to the } \\
\text { edge of the contour at } Q_{l}\end{array}$ \\
\hline$\hat{\mathbf{z}}_{\text {loc }}$ & u.v. tangent to the $\operatorname{rim}$ at $Q_{l}$, the local $\hat{\mathbf{z}}$ axis \\
\hline$\hat{\mathbf{r}^{\prime}}$ & $\begin{array}{c}\text { u. v. oriented from the source to the } \\
\text { integration point }\end{array}$ \\
\hline$\hat{\boldsymbol{\beta}}^{\prime}$ & u.v. parallel to the incidence plane \\
\hline$\hat{\phi}^{\prime}$ & u.v. perpendicular to the incidence plane \\
\hline$\hat{\mathbf{r}}$ & $\begin{array}{l}\text { u. v. oriented from the integration point to } \\
\text { the observation }\end{array}$ \\
\hline$\hat{\boldsymbol{\beta}}$ & u.v. parallel to the observation plane \\
\hline$\hat{\phi}$ & u.v. perpendicular to the observation plane \\
\hline
\end{tabular}

TABLE I

Geometrical QUANTITIES REQUiRed For THE APPLiCATION of THE ITD. "UNit VECTOR" IS ABBREVIATED WITH U.V.

At each point of the contour of a curved edge, one evaluates the incremental contributions by using a locally tangent wedge geometry. Referring to Fig. 2, let $S=$ $\left(x^{\prime}, y^{\prime}, z^{\prime}\right)$ be the source, $P=(x, y, z)$ the observation and $Q_{l}=\left(x_{l}, y_{l}, z_{l}\right)$ the diffraction point located over the rim of the obstacle. The geometrical quantities of interest are listed in Table I. Using these quantities, the angles for the coordinate system centered at the tangent point $Q_{l}$ are defined according to the following. The angle $\beta^{\prime}$ is computed as

$$
\beta^{\prime}=\arccos \left(\hat{\mathbf{r}}^{\prime} \cdot \hat{\mathbf{z}}_{\text {loc }}\right)
$$

and, similarly, the angle $\beta$ is

$$
\beta=\arccos \left(\hat{\mathbf{r}} \cdot \hat{\mathbf{z}}_{\text {loc }}\right) .
$$

Then, the angles $\phi^{\prime}$ and $\phi$ are computed as:

$$
\begin{aligned}
\phi^{\prime} & =\operatorname{atan} 2\left(-\hat{\mathbf{r}}^{\prime} \cdot \hat{\mathbf{y}}_{\mathbf{l o c}},-\hat{\mathbf{r}}^{\prime} \cdot \hat{\mathbf{x}}_{\text {loc }}\right), \\
\phi & =\operatorname{atan} 2\left(\hat{\mathbf{r}} \cdot \hat{\mathbf{y}}_{\mathbf{l o c}}, \quad \hat{\mathbf{r}} \cdot \hat{\mathbf{x}}_{\mathbf{l o c}}\right) .
\end{aligned}
$$




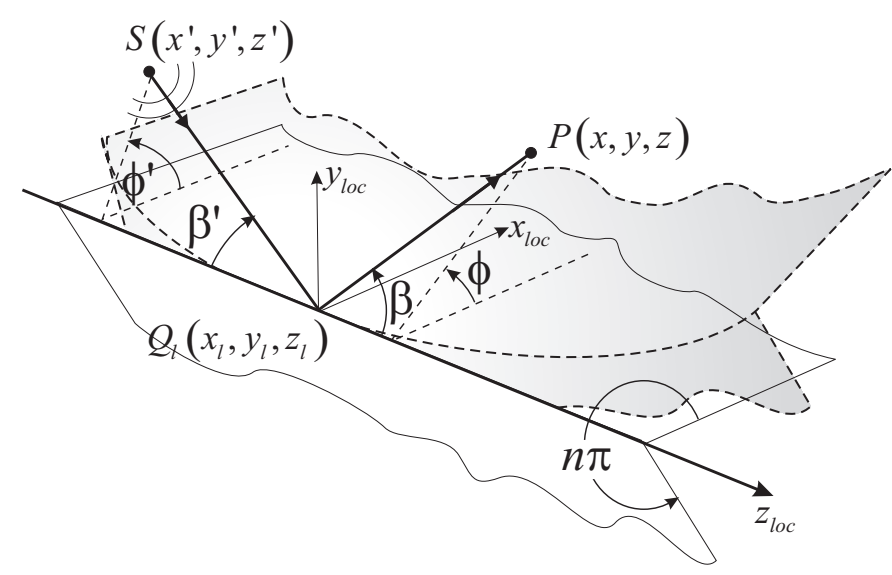

Fig. 2. Curved edge and reference system for the local tangent problem.

Notice that atan2 is the four quadrant inverse tangent, which returns an angle $0<\varphi<2 \pi$, and the negative signs are due to the fact that $\hat{\mathbf{r}}^{\prime}$ is oriented from $S$ to $Q_{l}$. Next, the function

$$
u\left(\beta^{\prime}, \beta\right)=\log \frac{(1-\cos \beta)\left(1+\cos \beta^{\prime}\right)}{\sin \beta^{\prime} \cdot \sin \beta} .
$$

is evaluated [19]. All previous quantities are required to calculate the scalar diffraction coefficients $F_{s}$ and $F_{h}$ defined as [1]

$$
\left\{\begin{array}{l}
F_{s}=\frac{1}{16 \pi^{2} n}\left(D_{1}+D_{2}-D_{3}-D_{4}\right) \\
F_{h}=\frac{1}{16 \pi^{2} n}\left(D_{1}+D_{2}+D_{3}+D_{4}\right)
\end{array}\right.
$$

where

$$
\left\{\begin{array}{l}
D_{1}=\frac{\sin \frac{\pi+\phi-\phi^{\prime}}{n}}{\cosh \frac{u}{n}-\cos \frac{\pi+\phi-\phi^{\prime}}{\phi^{\prime}}} \\
D_{2}=\frac{\sin \frac{\pi-\phi+\phi^{\prime}}{n}}{\cosh \frac{u}{n}-\cos \frac{\pi-\phi+\phi^{\prime}}{n}} \\
D_{3}=\frac{\sin \frac{\pi+\phi+\phi^{\prime}}{n}}{\cosh \frac{u}{n}-\cos \frac{\pi+\phi+\phi^{\prime}}{n}} \\
D_{4}=\frac{\sin \frac{\pi-\phi-\phi^{\prime}}{n}}{\cosh \frac{u}{n}-\cos \frac{\pi-\phi-\phi^{\prime}}{n}} .
\end{array}\right.
$$

The parameter $n$ is defined with reference to the exterior wedge angle $n \pi$ ( $n=\frac{3}{2}$ for the right-angle wedge case, $n=2$ for the disc case). The dyadic $\mathbf{D}(\mathbf{l})$ is then obtained as

$$
\mathbf{D}(\mathbf{l})=-\hat{\boldsymbol{\beta}}^{\prime} \hat{\boldsymbol{\beta}} F_{s}-\hat{\phi}^{\prime} \hat{\phi} F_{h},
$$

from which the vector incremental contribution is given by

$$
d \mathbf{E}(P)=-\left(F_{s} \mathbf{E}_{\mathbf{0}}(l) \cdot \hat{\boldsymbol{\beta}}^{\prime} \hat{\boldsymbol{\beta}}+F_{h} \mathbf{E}_{\mathbf{0}}(l) \cdot \hat{\boldsymbol{\phi}}^{\prime} \hat{\boldsymbol{\phi}}\right) \frac{e^{-j k r(l)}}{r(l)} .
$$

Finally, one can numerically integrate the quantity in (10) to compute the diffracted electric field given by (1).

\section{The Canonical PeC Straight Wedge PRoblem}

First, we compare the ITD with the UTD and measurement results for the PEC straight wedge. The UTD formulation used in this work is found in the original

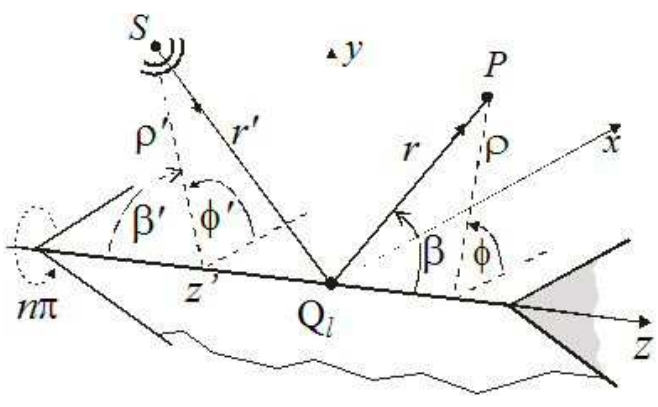

Fig. 3. Geometry of the Canonical PEC Straight Wedge Problem.

article by Kouyoumjian and Pathak [2] or in textbooks such as [20] and [21]. Fig. 3 shows the geometry of this problem, for which it is very well known that the UTD correctly evaluates the diffracted field, in particular at the reflection shadow boundary (RSB) and at the incident shadow boundary (ISB). The experiment, whose setup is shown in Fig. 4, is aimed at the measurement of the total electric field when the observation point crosses both the RSB and the ISB. The measurements were taken in the anechoic room of the Andrew Electromagnetics Laboratory at the University of Illinois at Chicago. The transmitter and receiver are identical horn antennas, operated at the frequency $f=25 \mathrm{GHz}$, for which the wavelength is $\lambda=1.2 \mathrm{~cm}$. The wedge is represented by a sheet of copper, with a straight edge obtained by bending the sheet to make an interior angle of $\pi / 2 \mathrm{rad}$. The edge corresponds to the $z$-axis in Fig. 4. During these measurements, the transmitter, Tx, is held at a constant position, with its main beam pointing towards the negative $x$-axis and polarized parallel to the $y$-axis. The receiver, $\mathrm{Rx}$, is moved vertically at increments of $\lambda / 10$, along the $y$-axis, so that it crosses both shadow boundaries. The distance from $\mathrm{Rx}$ to the edge of the wedge ( $\mathrm{z}$ axis) always exceeds the far-field distance of $\mathrm{Rx}$, as indicated in Fig 4 . During its movement, $\mathrm{Rx}$ is oriented so that its main beam is parallel to the positive $x$-axis and polarized parallel to the $y$-axis. In this experiment, care was taken to avoid wedge truncation effects, hence the absorbers were placed around the edges of the copper sheet. Both antennas are connected to a vector network analyzer HP 8510. The results shown in Fig. 5 report the $y$ component of the total electric field $\left|E_{y}^{t}\right|$, which is the superposition of the direct ray from $\mathrm{Tx}$ to $\mathrm{Rx}$, the ray reflected from the upper wedge face, and the ray diffracted by the straight edge. The ITD results were obtained following steps similar to the ones indicated in the previous section and specialized for the straight edge geometry. Additionally, in performing these evaluations, the radiation patterns of both antennas were taken into account by multiplying the incremental diffraction coefficient of eq. (10) by the measured radiation pattern function of the transmitter and the receiver. These radiation patterns were normalized so that their value is unity along the directions of maximum field strength. One observes that the ITD and the UTD results overlap very well, especially when crossing the RSB and the ISB 


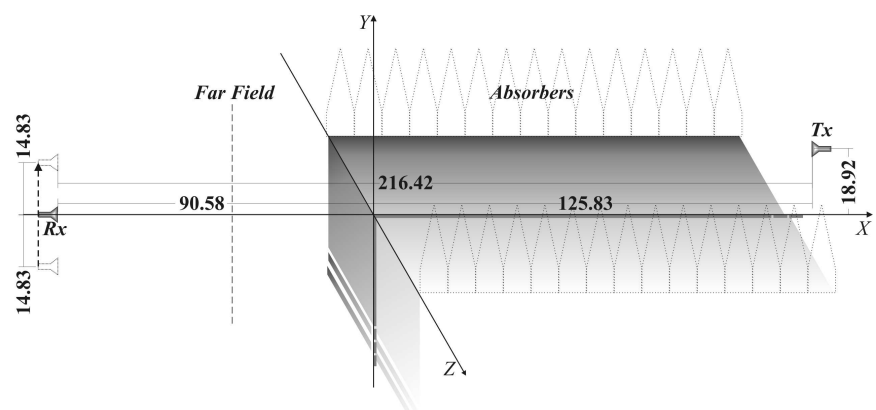

Fig. 4. PEC Straight Wedge Geometry. TX antenna located at $125.83 \lambda$ from the edge of the wedge and at a height of $18.92 \lambda$. RX antenna located at $90.58 \lambda$ from the edge of the wedge. Frequency $25 \mathrm{GHz}$. All distances in the figure are normalized to the wavelength $\lambda$.

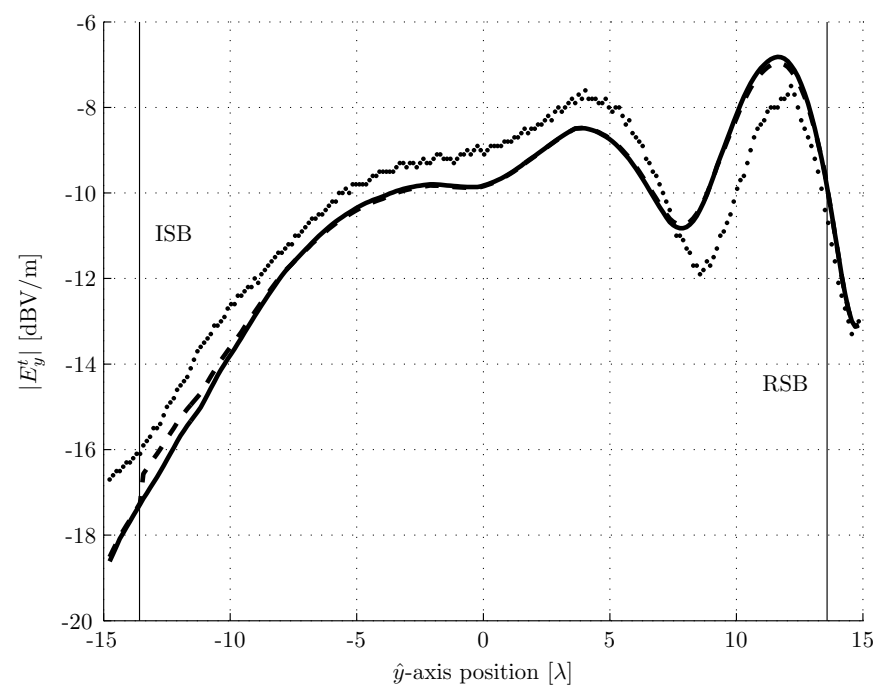

Fig. 5. Results of numerical evaluations and measurements for the Straight Wedge. Dashed Line: ITD. Continuous Line: UTD. Dots: Measurements. RSB located at $13.58 \lambda$, ISB located at $-13.58 \lambda$.

(indicated by the solid vertical lines). The measurement results also show a very good agreement, since the vertical divisions correspond to increment of only $2 \mathrm{~dB}$ each.

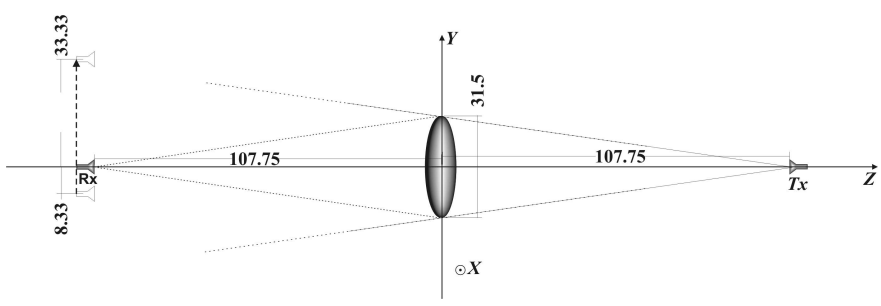

Fig. 6. PEC Circular Disc Geometry. Radius of the disc $15.75 \lambda$ thickness of the disc $0.25 \lambda$; distance of the antennas from disc center $107.75 \lambda$; frequency $25 \mathrm{GHz}$. All distances in the figure are normalized to the wavelength $\lambda$.

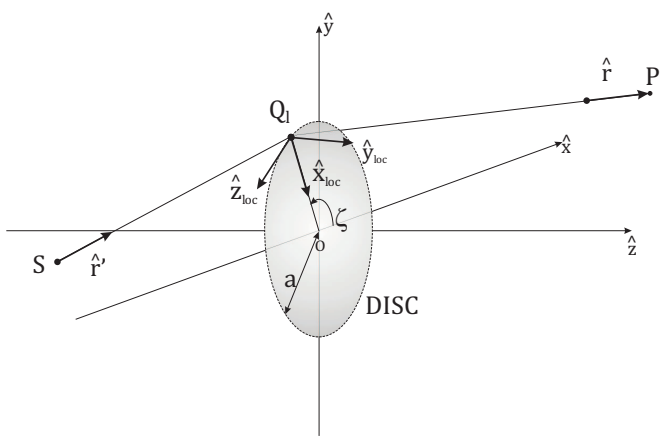

Fig. 7. Local reference system geometry for the ITD computation of the diffraction from a disc.

\section{The PeC Circular Disc Problem with ITD}

The second validation is the comparison of the ITD with measurements for the case of a PEC circular disc. This geometry is considered because it is challenging for a theory such as the UTD, due to the presence of caustic points along its axis. Fig. 6 shows the setup of the experiment, for which measurements were carried out at the frequency $f=25 \mathrm{GHz}$. A disc with diameter $d=0.378 \mathrm{~m}(d=31.5 \lambda)$ and thickness of $3 \mathrm{~mm}(\lambda / 4)$ is located in the $x y$ plane, with its center in the origin of the reference system. The disc is located at half-distance between Tx and Rx and is illuminated by a horn antenna, oriented with its main beam pointed towards the negative $z$ axis and polarized parallel to the $y$ axis. The receiver is a horn antenna, oriented with its main beam pointed towards the positive $z$ axis and polarized parallel to the $y$ axis. The receiver is moved vertically, parallel to the $y$ axis, at small increments corresponding to $\lambda / 10$ each. During the vertical movement, the receiver spans a length of $55 \mathrm{~cm}(45.83 \lambda)$, crosses the axis and the incident shadow boundary. For this geometry, ITD numerical evaluations were obtained following the steps described in Section II and using the quantities reported in Table II, which refer to Fig. 7. Additionally, in performing these evaluations, the radiation patterns of both antennas were taken into account as previously described for the case of the straight edge.

The results for the normalized $y$ component of the total received electric field $\left|E_{y}^{t}\right|$ are given in Fig. 8, which shows a very good agreement, especially when the receiver crosses the $z$-axis and the ISB. It should also be emphasized that the ITD formulation assumes an infinitesimally thin disc, while the disc used for the experiment is not thin at all since its thickness is $\lambda / 4$; nevertheless the agreement between measurements and the theoretical results is very good because the field is mainly due to forward scattering [22].

In the next section, further validations of the ITD are considered by comparing it with exact solutions. 


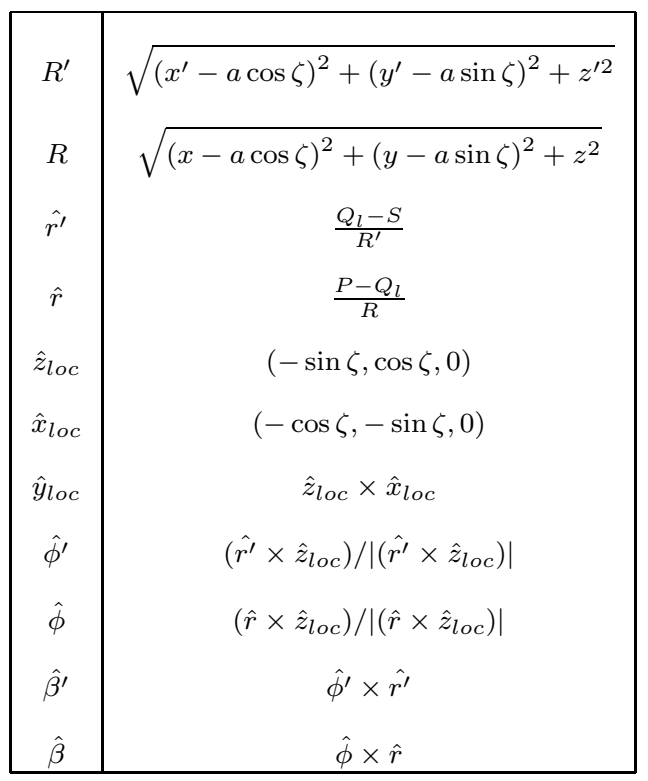

TABLE II

MATHEMATICAL EXPRESSIONS OF THE GEOMETRICAL QUANTITIES FOR THE GEOMETRY OF A DISC SHOWN IN Fig. 7

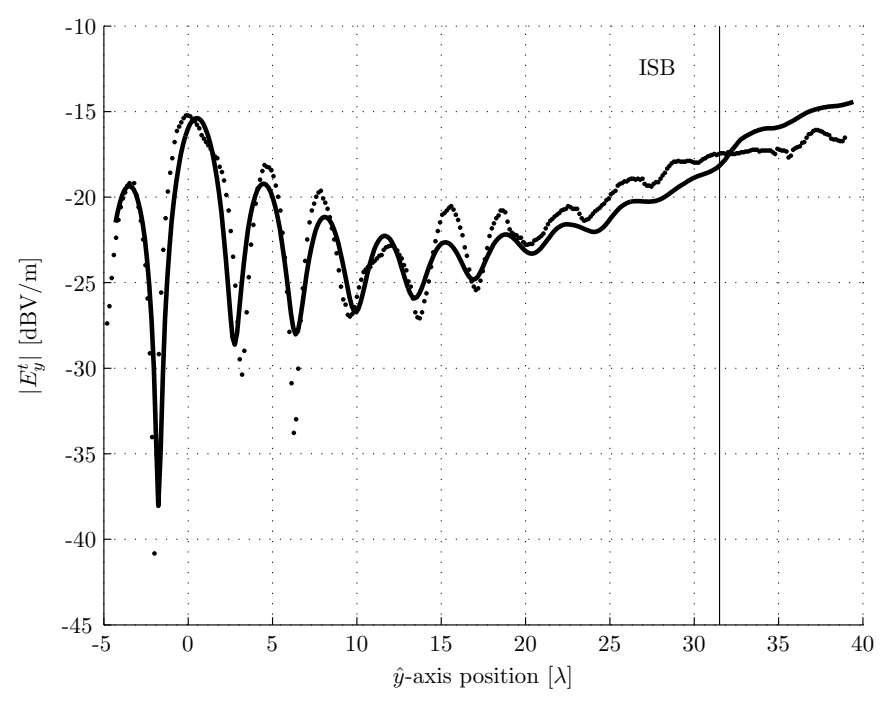

Fig. 8. Results of numerical evaluations and measurements for the PEC Disk. Continuous line: ITD. Dots: Measurements. ISB at $31.5 \lambda$

\section{VALIDATION WITH EXACT SOLUTIONS}

\section{A. Disc}

Exact analytical expressions to validate the ITD solutions are available in the special case that a PEC disc is illuminated by a dipole [23]. Other solutions exists and are listed in [24]. However, because the computation of the solution is cumbersome, we limit our attention to the special case of a dipole located along the axis of the disc and axially polarized [24], as shown in Fig. 9.

The disc geometry is a limiting case of an oblate spheroidal surface and, therefore, this problem is conveniently examined using the oblate spheroidal coordinates $(\eta, \xi, \varphi)$, which are related to the cartesian coordinates $(x, y, z)$ by:

$$
\left\{\begin{array}{l}
x=\frac{d}{2} \sqrt{\left(\xi^{2}+1\right)\left(1-\eta^{2}\right)} \cos \varphi \\
y=\frac{d}{2} \sqrt{\left(\xi^{2}+1\right)\left(1-\eta^{2}\right)} \sin \varphi \\
z=\frac{d}{2} \xi \eta
\end{array}\right.
$$

where $0 \leq \xi<\infty,-1 \leq \eta \leq 1,0 \leq \varphi \leq 2 \pi$, and $d$ is the focal distance.

The surface $\xi=$ constant is an oblate spheroid (i.e. a flattened ellipsoid of revolution) with major axis $d \sqrt{\xi^{2}+1}$ and minor axis $d \xi$, with $z$ as the symmetry axis.

The surface $|\eta|=$ constant is a hyperboloid of revolution with $z$ as the symmetry axis, $\eta>0(\eta<0)$ for $z>0$ $(z<0)$, and asymptotic cone of semi-aperture $\theta=\arccos \eta$; in particular, $|\eta|=1$ is the $z$-axis, whereas $\eta=0$ is the $z=0$ plane with the circle $\xi=0$ excluded. The surface $\varphi=$ constant is a half-plane originating in the $z$-axis. The surface $\xi=0$ is a disc with diameter $d$.

The inverse transformation from rectangular coordinates to oblate spheroidal coordinates is reported here for convenience:

$$
\left\{\begin{array}{l}
\varphi=\arctan \frac{y}{x} \\
\eta=\frac{z}{|z|} \sqrt{\frac{-\left[4\left(x^{2}+y^{2}+z^{2}\right)-d^{2}\right]+\sqrt{4\left[\left(x^{2}+y^{2}+z^{2}\right)-d^{2}\right]^{2}+16 d^{2} z^{2}}}{2 d^{2}}} \\
\xi=\sqrt{\frac{\left[4\left(x^{2}+y^{2}+z^{2}\right)-d^{2}\right]+\sqrt{4\left[\left(x^{2}+y^{2}+z^{2}\right)-d^{2}\right]^{2}+16 d^{2} z^{2}}}{2 d^{2}}} .
\end{array}\right.
$$

We assume as primary source an electric dipole located on the $z$-axis and axially oriented. For a primary electric source located on the $z$-axis and axially oriented, the electric and magnetic fields everywhere are of the type:

$$
\begin{array}{ll}
\mathbf{E}=E_{\xi}(\xi, \eta) \hat{\boldsymbol{\xi}}+E_{\eta}(\xi, \eta) \hat{\boldsymbol{\eta}}, & E_{\varphi}=0 \\
\mathbf{H}=H_{\varphi}(\xi, \eta) \hat{\boldsymbol{\varphi}}, & H_{\xi}=H_{\eta}=0
\end{array}
$$

where

$$
\begin{aligned}
& E_{\xi}=\frac{j Z}{c} \sqrt{\frac{1-\eta^{2}}{\xi^{2}+\eta^{2}}}\left(\frac{\partial}{\partial \eta}-\frac{\eta}{1-\eta^{2}}\right) H_{\varphi}, \\
& E_{\eta}=-\frac{j Z}{c} \sqrt{\frac{\xi^{2}+1}{\xi^{2}+\eta^{2}}}\left(\frac{\partial}{\partial \xi}+\frac{\xi}{\xi^{2}+1}\right) H_{\varphi},
\end{aligned}
$$

and $c=k d / 2$ is the product of the wavenumber and the interfocal radius, and $Z$ the intrinsic impedance of the medium.

An electric dipole located at $\left(\eta_{0}=1, \xi_{0}, 0\right) \equiv\left(0,0, z_{0}=\right.$ $\left.\xi_{0} d / 2\right)$ on the positive $z$-axis and axially oriented, with moment $\hat{\mathbf{z}} 4 \pi \varepsilon / k$ corresponding to an electric Hertz vector $\boldsymbol{\Pi}^{(e)}=\hat{\mathbf{z}} \exp (-j k R) /(k R)$ where $R$ is the distance of the observation point $(\eta, \xi, \phi)$ from the dipole, generates a primary magnetic field [24]:

$$
H_{\varphi}^{i}=-c k^{2} Y \frac{e^{-j k R}}{k^{2} R^{2}} \sqrt{\left(\xi^{2}+1\right)\left(1-\eta^{2}\right)}
$$

with $Y=1 / Z$. The incident magnetic field may be 
expressed as the following series expansion

$$
\begin{array}{r}
H_{\varphi}^{i}=\frac{2 k^{2} Y}{\sqrt{\xi_{0}^{2}+1}} \sum_{n=1}^{\infty} \frac{j^{n}}{\widetilde{\rho}_{1, n} \widetilde{N}_{1, n}} R_{1, n}^{(1)}\left(-j c, j \xi_{<}\right) \times \\
R_{1, n}^{(4)}\left(-j c, j \xi_{>}\right) S_{1, n}(-j c, \eta),
\end{array}
$$

where $R_{1, n}^{(1),(4)}$ are radial oblate spheroidal functions of the first and fourth kind, respectively, and $S_{1, n}$ are angular oblate spheroidal functions [25]. The quantity $\xi_{<}\left(\xi_{>}\right)$is the smaller (larger) between $\xi$ and $\xi_{0}$.

The total magnetic field is obtained by the superposition of the incident field (17) and the scattered field given by

$$
\begin{aligned}
& H_{\varphi}^{s}=-\frac{2 k^{2} Y}{\sqrt{\xi_{0}^{2}+1}} \sum_{n=1}^{\infty} \frac{j^{n}}{\widetilde{\rho}_{1, n} \widetilde{N}_{1, n}} \frac{R_{1, n}^{(1)^{\prime}}(-j c, j 0)}{R_{1, n}^{(4)^{\prime}}(-j c, j 0)} \times \\
& R_{1, n}^{(4)}\left(-j c, j \xi_{0}\right) R_{1, n}^{(4)}(-j c, j \xi) S_{1, n}(-i c, \eta) .
\end{aligned}
$$

The expression of the scattered field satisfies the radiation condition because of the presence of the oblate spheroidal function of the fourth kind $R_{1, n}^{(4)}(-j c, j \xi)$ as well as the boundary condition along the surface $\xi=0$ of the disc.

The total electric field is obtained by computing the appropriate partial derivatives of the magnetic field according to (14) and (15). Specifically, the components of the scattered electric field require

$$
\begin{aligned}
\frac{\partial H_{\varphi}^{s}}{\partial \xi}= & -\frac{2 k^{2} Y}{\sqrt{\xi_{0}^{2}+1}} \sum_{n=1}^{\infty} \frac{j^{n}}{\widetilde{\rho}_{1, n} \widetilde{N}_{1, n}} \frac{R_{1, n}^{(1)^{\prime}}(-j c, j 0)}{R_{1, n}^{(4)^{\prime}}(-j c, j 0)} \times \\
& R_{1, n}^{(4)}\left(-j c, j \xi_{0}\right) R_{1, n}^{(4)^{\prime}}(-j c, j \xi) S_{1, n}(-j c, \eta) \\
\frac{\partial H_{\varphi}^{s}}{\partial \eta}= & -\frac{2 k^{2} Y}{\sqrt{\xi_{0}^{2}+1}} \sum_{n=1}^{\infty} \frac{j^{n}}{\widetilde{\rho}_{1, n} \widetilde{N}_{1, n}} \frac{R_{1, n}^{(1)^{\prime}}(-j c, j 0)}{R_{1, n}^{(4)^{\prime}}(-j c, j 0)} \times \\
& R_{1, n}^{(4)}\left(-j c, j \xi_{0}\right) R_{1, n}^{(4)}(-j c, j \xi) S_{1, n}^{\prime}(-j c, \eta),
\end{aligned}
$$

while the same partial derivatives required for the computation of the incident electric field may be obtained directly from (16). In order to facilitate the comparison with the ITD results, we transform the oblate spheroidal components of the total electric field into cartesian components using the formulas:

$A_{x}=A_{\xi} \sqrt{\frac{1-\eta^{2}}{\xi^{2}+\eta^{2}}} \xi \cos \varphi+A_{\eta} \sqrt{\frac{1+\xi^{2}}{\xi^{2}+\eta^{2}}} \eta \cos \varphi-A_{\varphi} \sin \varphi$

$A_{y}=A_{\xi} \sqrt{\frac{1-\eta^{2}}{\xi^{2}+\eta^{2}}} \xi \sin \varphi+A_{\eta} \sqrt{\frac{1+\xi^{2}}{\xi^{2}+\eta^{2}}} \eta \sin \varphi+A_{\varphi} \cos \varphi$

$A_{z}=A_{\xi} \sqrt{\frac{1+\xi^{2}}{\xi^{2}+\eta^{2}}} \eta-A_{\eta} \sqrt{\frac{1-\eta^{2}}{\xi^{2}+\eta^{2}}} \xi$.

The exact expressions were computed using some of the Fortran numerical subroutines given by [26], which were modified by the authors. Additional details on the computations of the series (18)-(20), which require an acceleration method, are given in [27], [28], and [29]. For

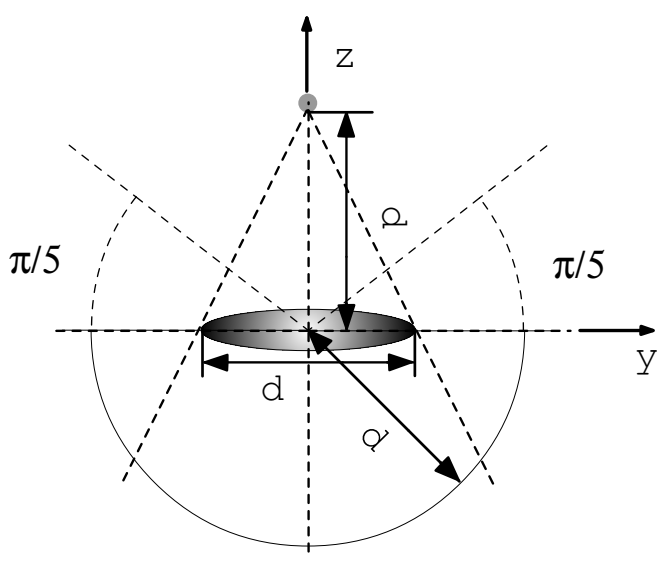

Fig. 9. Disc geometry for the validation using the exact solution.

the cases examined in this article, the series (18), (19), (20) converge within the first 20 terms. Fig. 10 and Fig. 11 show the results that were computed for a disc with diameter $d=20 \lambda$ and a dipole located along the positive $z$-axis at $z_{0}=20 \lambda$. These results represent two components of the total electric field computed along a line parallel to the $y$ axis, which intersects the $z$-axis at $z=-20 \lambda$. These results show an almost perfect overlap with the exact solution, thus increasing confidence in the use of the ITD. In fact, even though Figs. 10 and Fig. 11 only show comparisons among magnitudes, the perfect overlap is also observed among real and imaginary components of all the field, which are not reported here for brevity.

An even more interesting and challenging comparison is obtained by reducing the distance of the source and observation from the disc as well as by reducing the diameter of the disc. Fig. 12 shows the results obtained when the dipole source is located at $(0,0,5 \lambda)$ and the observation moves along the circumference of a circle located in the $x z$ plane, centered in the origin, and with radius $5 \lambda$. The disc radius is $2.5 \lambda$ and the angular parameter $\theta \in[-\pi / 5,6 \pi / 5]$, measured counter-clockwise with respect to the oblate spherical angle $\varphi$, identifies the observation point along the circumference, as shown in Fig. 9.

The agreement between ITD and the exact solution is very good, especially when one considers that (i) the radius of curvature of the disc is small; (ii) the source is only $5 \lambda$ away from the disc center; (iii) the observation is only $5 \lambda$ from the center of the disc; and, (iv) there is a smooth transition across $\theta=0$, i.e. for grazing aspect of observation. On the other hand, the UTD result for the same geometry exhibits a caustics when the observation point crosses the negative $\mathrm{z}$ axis at $\theta=\pi / 2$.

\section{B. Hole in a PEC plane}

As further validation, we consider a hole in a PEC ground plane, shown in Fig. 13, because this geometry allows to test the ITD behavior along a concave contour. This geometry is conveniently analyzed using the same 


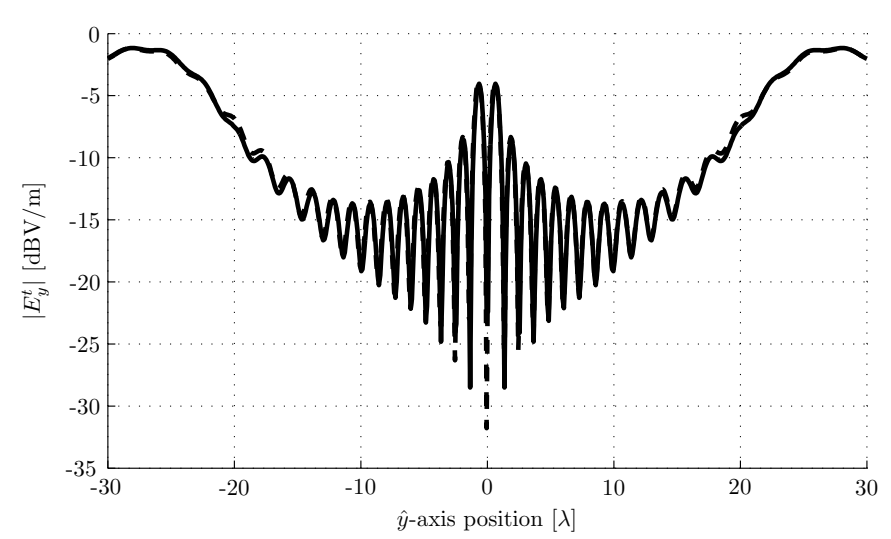

Fig. 10. Results of numerical and exact evaluations for the PEC Disk on a line $\left(d=20 \lambda, z_{0}=20 \lambda, z=20 \lambda\right)$. Continuous line: ITD. Dashed Line: Exact Solution.

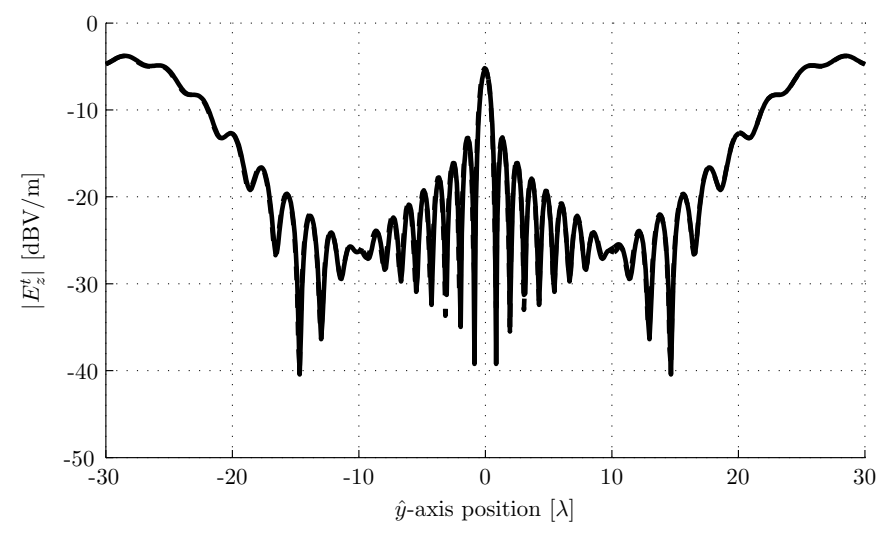

Fig. 11. Results of numerical and exact evaluations for the PEC Disk $\left(d=20 \lambda, z_{0}=20 \lambda, z=20 \lambda\right)$. Continuous line: ITD. Dashed Line: Exact Solution.

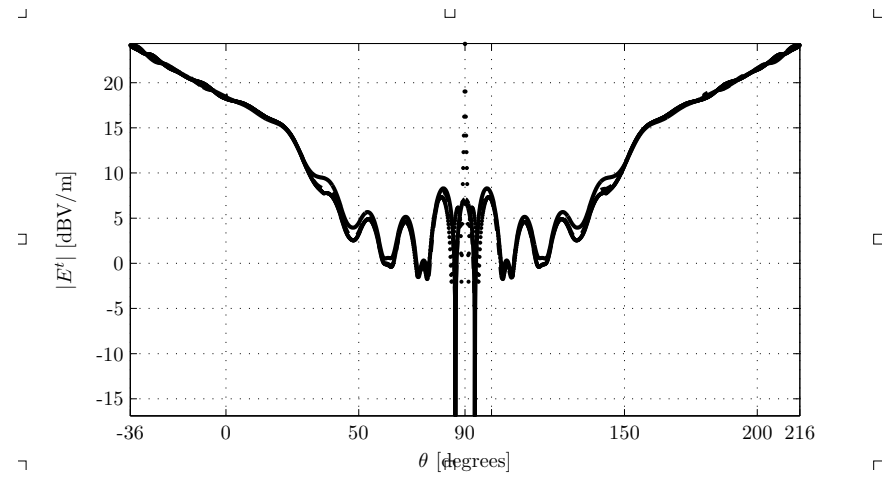

Fig. 12. Diffraction from a PEC disk of diameter $5 \lambda$ evaluated along an arch of circumference of radius $5 \lambda$ for a dipole at $z_{0}=5 \lambda$ using ITD, UTD and exact solutions. The plot shows: exact solution (continuous line); UTD solution (dotted line); and, ITD solution (dashed line).

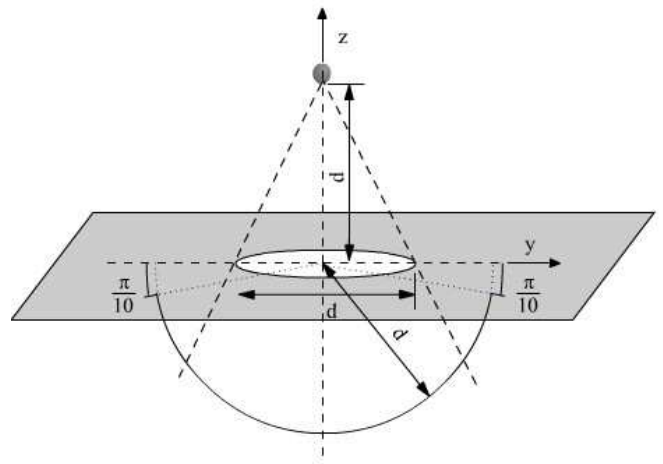

Fig. 13. Geometry for a hole in a PEC plane for the validation using the exact solution.

spheroidal coordinate system adopted for the disc geometry. The source is the electric dipole already considered for the disc geometry, so that the incident field is still represented by (16) or (17). Incidentally, this is not the complementary problem according to Babinet because the source is the same. The expression for the total magnetic field $H_{\varphi}$ when $z<0$ may be obtained following a procedure similar to the one indicated in [27]. In particular, $H_{\varphi}$ is given by the complex conjugate of (29) of [27]

$$
\begin{aligned}
& H_{\varphi}=\frac{2 j k^{2} Y}{\sqrt{\xi_{0}^{2}+1}} \sum_{l=0}^{\infty} \frac{(-1)^{l}}{\widetilde{\rho}_{1,2 l+1} \widetilde{N}_{1,2 l+1}} \frac{R_{1,2 l+1}^{(1)}(-j c, j 0)}{R_{1,2 l+1}^{(4)}(-j c, j 0)} \times \\
& R_{1,2 l+1}^{(4)}\left(-j c, j \xi_{0}\right) R_{1,2 l+1}^{(4)}(-j c, j \xi) S_{1,2 l+1}(-j c, \eta) .
\end{aligned}
$$

and the electric field components are still obtained from Maxwell equations using (14) and (15). For the case considered in this paper, series (24) converges within the first 20 terms.

Referring to Fig. 13, when the dipole is located at $z_{0}=20 \lambda$, with a hole diameter $d=20 \lambda$ and an observation circular arch with radius $20 \lambda$ the results for the total electric field $\left|E^{t}\right|$ are shown in Fig. 14 and indicate a very good agreement between the ITD and exact solutions.

The comparison is repeated for a more challenging case with $z_{0}=5 \lambda$, hole diameter $d=5 \lambda$ and radius of the observation circular arch $5 \lambda$. The results, shown in Fig. 15, indicate an overall good agreement, with the largest difference at the caustic.

\section{Conclusions}

This paper presented independent methods to validate the single diffraction ITD in the case of the geometry of the straight wedge, the circular disc, and a hole in a plane. This paper is important because it shows, for the first time, comparisons of the ITD with measurement results. It also shows a validation by a comparison with exact analytical formulations of the diffraction by a disc and a hole in a plane due to a dipole located along the axis of symmetry. This paper considered the first order formulation of the ITD. Specifically, we validated the locality principle that 


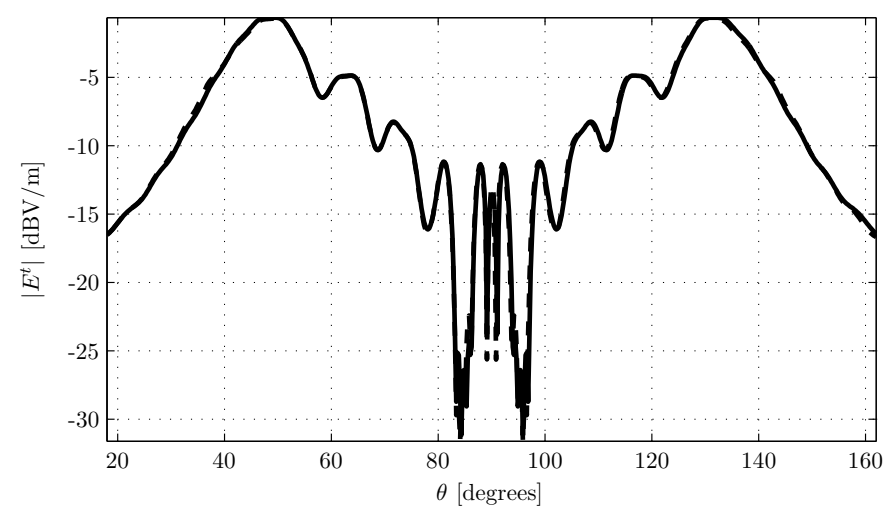

Fig. 14. Results of numerical and exact evaluations for the hole with a $20 \lambda$ diameter on an arch of circumference for a dipole at $z_{0}=20 \lambda$. Dashed line: ITD. Continuous line: Exact solution.

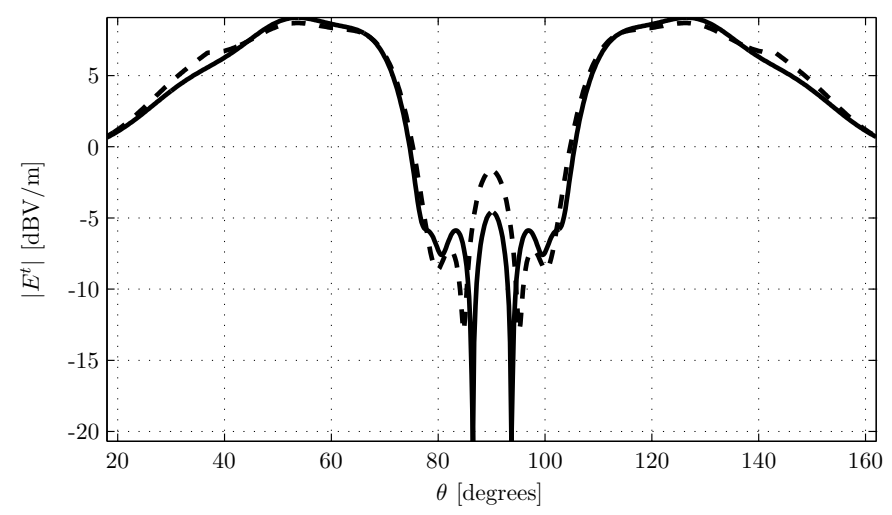

Fig. 15. Results of numerical and exact evaluations for the hole with a $5 \lambda$ diameter on an arch of circumference for a dipole at $z_{0}=5 \lambda$. Dashed line: ITD. Continuous line: Exact solution.

is at the foundation of the ITD and that enables the description of a curved edge starting from the solution of the straight edge local canonical problem. Finally, this paper contributes by providing a simple recipe for the application of the ITD.

Considerations related to the behavior of the single diffraction ITD at grazing aspect of incidence were made by Shore and Yaghjian in [30]. The single diffraction ITD shares a limitation that is common among ray-based methods in that it cannot properly describe physical situations that involve interactions among edges. Such interactions occur, for example, at grazing incidence on finite structures [31]. To correctly describe these interactions, one approach consists of including higher order diffraction mechanisms, such as the double diffraction mechanism for the UTD described in [32], [33] and [34]. Let us consider what happens in the case of the disc. At each point $Q_{l}$ along the rim of the disc, the ITD considers the locally tangent wedge geometry, shown in Fig. 2, which is a halfplane that extends to infinity in the direction of $\hat{\boldsymbol{x}}_{\mathbf{l o c}}$. Therefore, no interactions between $Q_{l}$ and other points along the rim of the disc are accounted for by this locally tangent geometry. At grazing incidence, however, it is important to include the contribution of interactions between diametrically opposite points and this contribution is not included in the single diffraction ITD, but it appears in the double diffraction ITD where two locally tangent geometry are simultaneously considered. Hence, to correctly describe diffracted fields at grazing incidence within the ITD framework, one must consider at least the double diffraction mechanism that is discussed in [35], but is beyond the purpose of this paper. In any case, for the disc, observation at grazing aspect is correctly evaluated by the single diffraction ITD, as it is apparent from Fig. 12 for values of the observation angle around $0^{\circ}$.

Application of the ITD should be considered when evaluating diffraction from curvilinear edges that belong to plane surfaces because all one has to do is simply to carry out a numerical evaluation of an integral, without the need to explicitly consider the location of points of stationary phase. As shown in this paper, the ITD does not suffer from discontinuities at caustics and provides correct results.

\section{ACKNOWLEDGEMENTS}

The authors would like to thank Mr. Giuseppe Carluccio and Mr. Lorenzo Lo Monte for their help with the figures. The authors are also thankful to the Reviewers for their useful comments.

\section{REFERENCES}

[1] R. Tiberio, A. Toccafondi, A. Polemi, and S. Maci, "Incremental Theory of Diffraction: a New-Improved Formulation," IEEE Trans. Antennas Propagat., vol. 52, no. 9, pp. 2234-2243, Sept. 2004.

[2] R. G. Kouyoumjian and P. H. Pathak, "A uniform geometrical theory of diffraction for an edge in a perfectly conducting surface," Proc. IEEE, vol. 62, no. 11, pp. 1448-1461, Nov 1974.

[3] J. B. Keller, "Geometrical Theory of Diffraction," J. Opt. Soc. Am, vol. 52, pp. 116-130, 1962.

[4] P. Y. Ufimtsev, "Method of edge waves in the physical theory of diffraction," Tech. Rep., U.S. Air Force Foreign Technology Division, Wright-Patterson AFB, OH, Sept. 71971.

[5] K. M. Mitzner, "Incremental length diffraction coefficients," Tech. Rep. AFAL-TR-73-296, Northrop Corporation, Aircraft Division, April 1974.

[6] A. Michaeli, "Equivalent edge currents for arbitrary aspects of observation," IEEE Trans. Antennas Propagat., vol. 32, pp. 252258, March 1984.

[7] A. Michaeli, "Elimination of infinities in equivalent edge currents - I: Fringe current components," IEEE Trans. Antennas Propagat., vol. 34, pp. 912-918, July 1986.

[8] R. A. Shore and A. D. Yaghjian, "Incremental diffraction coefficients for planar surfaces," IEEE Trans. Antennas Propagat., vol. 36, no. 1, pp. 55-70, Jan. 1988.

[9] D. I. Butorin and P. Y. Ufimtsev, "Explicit expressions for an acoustic edge wave scattered by an infinitesimal edge element," Sov. Phys. Acoust, vol. 32, no. 4, pp. 283-287, July-Aug. 1986.

[10] P. Y. Ufimtsev, "Elementary edge waves and the physical theory of diffraction," Electromagnetics, vol. 11, no. 2, pp. 125-159, April-June 1991.

[11] R. A. Shore and A. D. Yaghjian, "Application of incremental length diffraction coefficients to calculate the pattern effects of the rim and surface cracks of a reflector antenna," IEEE Trans. Antennas Propagat., vol. 41, no. 1, pp. 1-11, Jan. 1993.

[12] R. A. Shore and A. D. Yaghjian, "Shadow boundary incremental length diffraction coefficients applied to scattering from 3-D bodies," IEEE Trans. Antennas Propagat., vol. 49, no. 2, pp. 200-210, Feb. 2001. 
[13] A. D. Yaghjian, "Incremental length diffraction coefficients for arbitrary cylindrical scatterers," IEEE Trans. Antennas Propagat., vol. 49, no. 7, pp. 1025-1032, July 2001.

[14] R. Tiberio, S. Maci, and A. Toccafondi, "An incremental theory of diffraction: Scalar formulation," IEEE Trans. Antennas Propagat., vol. 42, no. 5, pp. 600-612, May 1994.

[15] R. Tiberio, S. Maci, and A. Toccafondi, "An incremental theory of diffraction: Electromagnetic formulation," IEEE Trans. Antennas Propagat., vol. 43, no. 1, pp. 87-97, Jan. 1995.

[16] S. Maci, R. Tiberio, and A. Toccafondi, "Incremental diffraction coefficients for source and observation at finite distance from an edge," IEEE Trans. Antennas Propagat., vol. 44, 1996.

[17] R. Tiberio, S. Maci, and A. Toccafondi, "An incremental theory of diffraction for objects with local cylindrical shape," Transaction on IEICE, vol. E-80, no. 11, pp. 1367-1373, Nov. 1997, Special issue on Electromagnetic theory - Scattering and Diffraction.

[18] J. H. Meloling and R. J. Marhefka, "A caustic corrected UTD solution for the fields radiated by a source on a flat plate with a curved edge," IEEE Trans. Antennas Propagat., vol. 45, no. 12, pp. 1839-1849, Dec. 1997.

[19] A. Rubinowicz, "The Miyamoto-Wolf Diffraction Wave," Progress in Optics, vol. 4, pp. 331-377, 1965.

[20] C. A. Balanis, Advanced Engineering Electromagnetics, Wiley, New York, 1989.

[21] D. A. McNamara, C. W. I. Pistorius, and J. A. G. Malherbe, Introduction to the Uniform Geometrical Theory of Diffraction, Artech House, Boston - London, 1990.

[22] J. L. Volakis and M. A. Ricoy, "Diffraction by a thick perfectly conducting half-plane," IEEE Trans. Antennas Propagat., vol. 35 , no. 1 , pp. $62-72$, Jan. 1987.

[23] J. Meixner, "Theorie der Beugung elektromagnetischer Wellen an der vollkommen leitenden Kresscheibe und verwandte Probleme," Ann. Physik, vol. 12, pp. 227-236, 1953.

[24] J. J. Bowman, T. B. A. Senior, and P. L. E. Uslenghi, Electromagnetic and Acoustic Scattering by Simple Shapes, Hemisphere Publishing Corporation, New York, 1987.

[25] C. Flammer, Spheroidal wave functions, Stanford University Press, 1957.

[26] S. Zhang and J.-M. Jin, Computation of Special Functions, Wiley, New York, 1996.

[27] C. Berardi, D. Erricolo, and P. L. E. Uslenghi, "Exact dipole radiation for an oblate spheroidal cavity filled with isorefractive material and aperture-coupled to a half space," IEEE Trans. Antennas Propagat., vol. 52, no. 9, pp. 2205-2213, Sept. 2004.

[28] D. Erricolo and P. L. E. Uslenghi, "Exact Radiation for Dipoles on Metallic Spheroids at the Interface Between Isorefractive Half-Spaces," IEEE Trans. Antennas Propagat., vol. 53, no. 12, pp. 3974-3981, Dec. 2005.

[29] D. Erricolo, "Acceleration of the convergence of series containing Mathieu functions using Shanks transformation," IEEE Antennas Wireless Propagat. Lett., vol. 2, pp. 58-61, 2003.

[30] R. A. Shore and A. D. Yaghjian, "A comparison of highfrequency scattering determined from PO fields enhanced with alternative ILDCs," IEEE Trans. Antennas Propagat., vol. 52, no. 1, pp. 336-341, Jan. 2004.

[31] R. Tiberio and R. G. Kouyoumjian, "A uniform GTD solution for the diffraction by strips illuminated at grazing incidence," Radio Sci., vol. 14, pp. 933-941, 1979.

[32] M. Albani, "A uniform double diffraction coefficient for a pair of wedges in arbitrary configuration," IEEE Trans. Antennas Propagat., vol. 53, no. 2, pp. 702-710, Feb. 2005.

[33] M. Albani, F. Capolino, S. Maci, and R. Tiberio, "Diffraction at a thick screen including corrugations on the top face," IEEE Trans. Antennas Propagat., vol. 45, no. 2, pp. 277-283, Feb. 1997.

[34] F. Capolino, M. Albani, S. Maci, and R. Tiberio, "Double diffraction at a pair of coplanar skew edges," IEEE Trans. Antennas Propagat., vol. 45, no. 8, pp. 1219-1226, Aug 1997.

[35] A. Toccafondi and R. Tiberio, "An incremental theory of double edge diffraction," Radio Sci., vol. 42, no. 6, Dec. 2007, Article Number RS6S30, DOI: 10.1029/2007RS003681.

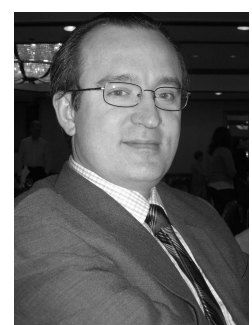

Danilo Erricolo (S'97-M'99-SM'03) received the Laurea degree of Doctor in electronics engineering (summa cum laude) from Politecnico di Milano, Milan, Italy, in 1993 and the Ph.D. degree in electrical engineering and computer science from the University of Illinois at Chicago (UIC), in 1998.

$\mathrm{He}$ is an Associate Professor in the Electrical and Computer Engineering Department, UIC, where he also is Associate Director of the Andrew Electromagnetics Laboratory. His research interests are primarily in the areas of wireless communications, electromagnetic scattering, and electromagnetic compatibility. His research activity is supported by the National Science Foundation and the Department of Defense. He has authored or coauthored more than 100 publications in refereed journals and international conferences.

Dr. Erricolo is a Member of Eta Kappa $\mathrm{Nu}$ and was elected a Full Member of the United States National Committee of the International Union of Radio Science (USNC-URSI) Commission B and E. He serves as Vice-Chair for the USNC-URSI Commission E. He was twice awarded both the Andrew Foundation Fellowship and the Beltrami Foundation Fellowship. He is an Associate Editor for the IEEE ANTENNAS AND WIRELESS PROPAGATION LETTERS. He also has served as Vice Chair of the Local Organizing Committee of the XXIX URSI General Assembly and has been appointed as General Chair of the 2012 IEEE Antennas and Propagation International Symposium/USNC National Radio Science Meeting in Chicago, IL, USA.

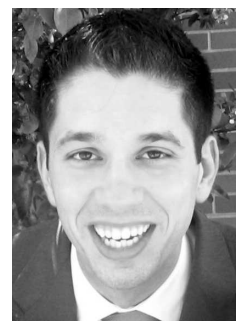

Stefano M. Canta (S'06-) received the Laurea degree in Telecommunications Engineering in 2004 and the Laurea Specialistica degree in Telecommunications Engineering in 2007 from Politecnico di Milano, Milan, Italy. Since August 2005 he has been a Ph.D. candidate in the Department of Electrical and Computer Engineering of the University of Illinois at Chicago. His research interests are in applied electromagnetism, specifically propagation and highfrequency methods. He is the recipient of the 2007 Andrew Fellowship.

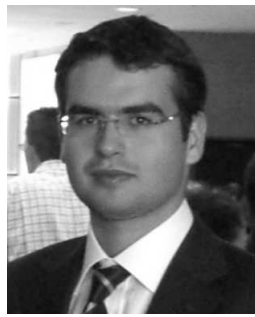

Harun T. Hayvaci (S'01-) received the Bachelor of Science degree in Electrical and Electronics Engineering from Bilkent University, Ankara, Turkey. Since August 2005 he has been a Ph.D. candidate in the Department of Electrical and Computer Engineering of the University of Illinois at Chicago. His research interests are in applied electromagnetism on antenna design and propagation models on scattering rich environment for radar and communication applications. 


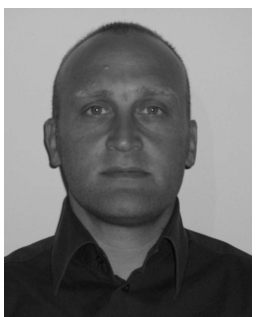

Matteo Albani (M'98) was born in Florence, Italy, on January 5, 1970. He received the Laurea degree in Electronic Engineering and the Ph.D. in Telecommunications Engineering from the University of Florence, Italy, in 1994 and 1998, respectively. From 1999 to 2001, he was an Associate Researcher with the Department of Information Engineering, University of Siena, Siena, Italy. In 2001, he joined the University of Messina, Messina, Italy, as an Assistant Professor at the Engineering Faculty. Since 2005 he moved back to the University of Siena where he is an Adjunct Professor. His research interests encompass highfrequency methods for electromagnetic scattering and propagation, numerical methods for array antennas, antenna analysis and design. Dr. Albani was awarded the "Giorgio Barzilai" prize for the Best Young Scientist paper at the Italian National Conference on Electromagnetics in 2002 (XIV RiNEm). 